\title{
Non-geometric convergence of the classical alternating Schwarz method
}

\author{
Gabriele Ciaramella, Richard M. Höfer
}

\section{Introduction}

Let $\Omega$ be a domain in $\mathbb{R}^{n}$ and $f \in L^{2}(\Omega)$ be a given function. Consider the Laplace problem

$$
\Delta u=f \text { in } \Omega, \quad u=0 \text { on } \partial \Omega .
$$

In error form, the alternating Schwarz method for the solution to (1) is

$$
\begin{aligned}
\Delta e_{1}^{n} & =0 \quad \text { in } \Omega_{1}, & \Delta e_{2}^{n} & =0 \text { in } \Omega_{2}, \\
e_{1}^{n} & =0 \quad \text { on } \partial \Omega \cap \bar{\Omega}_{1}, & e_{2}^{n} & =0 \text { on } \partial \Omega \cap \bar{\Omega}_{2}, \\
e_{1}^{n} & =e_{2}^{n-1} \text { on } \Gamma_{1}, & e_{2}^{n} & =e_{1}^{n} \text { on } \Gamma_{2} .
\end{aligned}
$$

Given any initial guess $e_{0} \in V:=H_{0}^{1}(\Omega)$ and solving iteratively (2), one obtains the sequence $\left(e_{1}^{n}\right)_{n \in \mathbb{N}^{+}} \subset H^{1}\left(\Omega_{1}\right)$ of errors in $\Omega_{1}$ and the sequence $\left(e_{2}^{n}\right)_{n \in \mathbb{N}^{+}} \subset H^{1}\left(\Omega_{2}\right)$ of errors in $\Omega_{2}$. Let us define the sequence $\left(e_{k}\right)_{k \in \mathbb{N}^{+}} \subset V$ as

$e_{k}:=\left\{\begin{array}{ll}e_{1}^{k} & \text { in } \bar{\Omega}_{1} \\ e_{2}^{k-1} & \text { in } \bar{\Omega} \backslash \Omega_{1}\end{array}\right.$ for $k$ odd, $\quad$ and $\quad e_{k}:=\left\{\begin{array}{ll}e_{2}^{k} & \text { in } \bar{\Omega}_{2} \\ e_{1}^{k-1} & \text { in } \bar{\Omega} \backslash \Omega_{2}\end{array}\right.$ for $k$ even.

We denote by $V_{1}$ and $V_{2}$ the extensions by zero in $\Omega$ of $H_{0}^{1}\left(\Omega_{1}\right)$ and $H_{0}^{1}\left(\Omega_{2}\right)$. Their orthogonal complements $V_{1}^{\perp}$ and $V_{2}^{\perp}$ in $V$ with respect to the inner product $\langle\cdot, \cdot\rangle:=$ $(\nabla \cdot, \nabla \cdot)_{L^{2}}$ are of the form

$$
V_{j}^{\perp}=\left\{v \in H_{0}^{1}(\Omega): \Delta v=0 \text { in } \Omega \backslash \overline{\Omega_{j}}\right\}
$$

G. Ciaramella

Universität Konstanz, Germany, e-mail: gabriele.ciaramella@uni-konstanz.de

R. M. Höfer

University of Bonn, Germany, e-mail: hoefer@iam.uni-bonn.de 
for $j=1,2$. It is then possible to show that (2) is equivalent to the alternating projection method (APM), $e_{k}:=P_{V_{2}^{\perp}} P_{V_{1}^{\perp}} e_{k-1}$, for $k \in \mathbb{N}^{+}$, where $P_{V_{j}^{\perp}}$ denote the orthogonal projections onto $V_{j}^{\perp}, j=1,2 ;[11,5]$.

For an arbitrary Hilbert space $V$ and two closed subspaces $V_{1}$ and $V_{2}$, von Neumann [12] and Halperin [10] proved that $e_{k} \rightarrow 0$ whenever $\overline{V_{1}+V_{2}}=V$. Moreover, if $V_{1}+V_{2}$ is closed, then the convergence is geometric, i.e. there exists $\theta<1$ such that for all $e_{0} \in V$ it holds that $\left\|e_{k}\right\| \leq \theta^{k}\left\|e_{0}\right\|$. In the particular case of only two subspaces $V_{1}$ and $V_{2}$, it is proven that the optimal $\theta$ is $\operatorname{incl}\left(V_{1}, V_{2}\right)$, with $0 \leq \operatorname{incl}\left(V_{1}, V_{2}\right) \leq 1$ the inclination between the subspaces $V_{1}$ and $V_{2}$, and that $\theta=\operatorname{incl}\left(V_{1}, V_{2}\right)<1$ if and only if $V_{1}+V_{2}$ is closed; see, e.g., [6, 5].

In the context of Schwarz method, P.L. Lions proves in [11] that an overlapping decomposition $\Omega=\Omega_{1} \cup \Omega_{2}$ guarantees that $\overline{V_{1}+V_{2}}=V$, and gives sufficient conditions for $V_{1}+V_{2}=\bar{V}_{1}+V_{2}$ to hold; see also [5, Lemma 2.16 and Theorem 2.17]. These conditions hold if the overlap $\Omega_{1} \cap \Omega_{2}$ is a sufficiently regular domain. A natural question arises: what happens if $\Omega_{1} \cap \Omega_{2}$ is not regular enough (e.g., nonLipschitz)? Is the geometric convergence still guaranteed in this case?

We show in this paper that if $\Omega_{1} \cap \Omega_{2}$ is non-Lipschitz, then $V_{1}+V_{2}$ is not necessarily closed. Classical abstract results state that in this case the APM converges 'arbitrarily slowly' [7, 8, 1]:

Definition 1 (Arbitrarily slow convergence (ASC)). The APM is said to converge arbitrarily slowly if for every sequence $\left(f_{n}\right)_{n \in \mathbb{N}} \subset \mathbb{R}_{+}$with $f_{n} \rightarrow 0$ and for all $\varepsilon>0$ there exists $e_{0} \in V$ with $\left\|e_{0}\right\|<\sup _{n} f_{n}+\varepsilon$ and $\left\|e_{k}\right\| \geq f_{n}$ for all $n$.

An ASC is quite difficult to observe and characterize. Therefore, we introduce the notion of 'non-geometric' convergence:

Definition 2 (Non-geometric convergence (NGC)). The APM is said to converge non-geometrically if there is no $\theta<1$ such that for all $e_{0} \in V$ it holds that $\left\|e_{k}\right\| \leq$ $\theta^{k}\left\|e_{0}\right\|$. Moreover, we say that a vector $e_{0} \in V$ leads to NCG, if there exists no $\theta<1$ such that $\left\|e_{k}\right\| \leq \theta^{k}\left\|e_{0}\right\|$.

To the best of our knowledge, the case of a non-closed sum $V_{1}+V_{2}$ is not studied in the literature of classical Schwarz theory. Moreover, also the literature concerning the more general framework of the APM presents surprisingly few results for this problem. The aim of our work is to study ASC and NGC of the classical Schwarz method and hence to shed more light on the issue of 'slow convergence' of the APM. To do so, in Section 2 we present a domain decomposition example that leads to two subspaces $V_{1}$ and $V_{2}$ whose sum is not closed. Section 3 focuses on theoretical results about NGC and ASC of the APM. In Section 4, we consider again the example from Section 2 and discuss the dependence of the convergence rate on the initial function $e_{0}$. Moreover, we precisely characterize a dense subset of the set of all functions leading to geometric convergence. Finally, results of numerical experiments are presented in Section 5. 


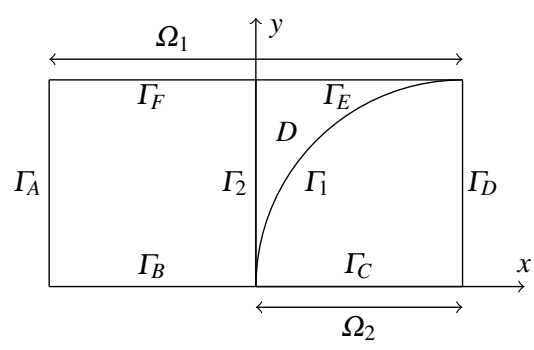

Fig. 1 Decomposition $\Omega=\Omega_{1} \cup \Omega_{2}$ with $D=\left\{(x, y) \in \Omega: x>0, y>x^{\alpha}\right\}=\Omega_{1} \cap \Omega_{2}$ and $\alpha<1$.

\section{Domain decomposition with non-Lipschitz overlap}

Consider a domain $\Omega=(-1,1) \times(0,1)$ and two subdomains $\Omega_{1}=(-1,0] \times(0,1) \cup$ $D$ and $\Omega_{2}=(0,1) \times(0,1)$ with $D=\left\{(x, y) \in \Omega: x>0, y>x^{\alpha}\right\}$ for some $\alpha>0$. Clearly, the overlapping decomposition $\Omega_{1} \cup \Omega_{2}=\Omega$ holds, and $D$ is the overlap; see Fig. 1. The following theorem shows that, if $\alpha<1$ (hence $D$ is a non-Lipschitz domain), then the decomposition $\Omega=\Omega_{1} \cup \Omega_{2}$ leads to two subspaces $V_{1}$ and $V_{2}$ of $V$ whose sum is not closed.

Theorem 1 (Non-closedness of $\mathbf{V}_{\mathbf{1}}+\mathbf{V}_{\mathbf{2}}$ ). Let $V_{j}$ denote the extension by zero in $\Omega$ of $H_{0}^{1}\left(\Omega_{j}\right)$ for $j=1,2$. Then $\overline{V_{1}+V_{2}}=H_{0}^{1}(\Omega)$, but $V_{1}+V_{2} \neq V$ for any $\alpha<1$.

Proof. Let $v \in{\overline{V_{1}+V_{2}}}^{\perp}$. Then $v \in V_{j}^{\perp}$ (see (3)), for $j=1$, 2. In particular $\Delta v=0$ in $\Omega$, thus $v=0$. This proves that $\overline{V_{1}+V_{2}}{ }^{\perp}=\{0\}$ and the first claim follows.

To prove the second statement, we consider the function $v=\left(r^{\beta} \sin \phi\right) \psi$, where $(r, \phi)$ denote polar coordinates and $\psi \in C^{1}(\bar{\Omega})$ is a cut-off function with $\psi=0$ on $\partial \Omega \backslash\{y=0\}$ and $\psi=1$ in $\left[-2^{-\alpha^{-1}}, 2^{-\alpha^{-1}}\right] \times\left[0, \frac{1}{2}\right]$. A direct calculation shows that $v \in H_{0}^{1}(\Omega)$ for $\beta>0$, and we now prove that $v \notin \overline{V_{1}+V_{2}}$. To do so, assume for the sake of contradiction that there are $v_{1} \in V_{1}$ and $v_{2} \in V_{2}$ such that $v=v_{1}+v_{2}$. Clearly, it must hold that $v_{1}=v$ on $\{x=0\}$ and $v_{1}=0$ on $\left\{\left(x, x^{\alpha}\right): 0 \leq x \leq 1\right\}$. Let $\gamma(y):=y^{\alpha^{-1}}$. Then $v_{1}(\gamma(y), y)=0$ and we get $-v_{1}(0, y)=\int_{0}^{\gamma(y)} \partial_{x} v_{1}(t, y) d t .^{1}$ Hence, we have

$$
\begin{aligned}
\left\|\nabla v_{1}\right\|_{L^{2}}^{2} & \geq \int_{0}^{\frac{1}{2}} \int_{0}^{\gamma(y)}\left|\partial_{x} v_{1}(t, y)\right|^{2} d t d y \geq \int_{0}^{\frac{1}{2}}\left[\int_{0}^{\gamma(y)} \partial_{x} v_{1}(t, y) d t\right]^{2} \frac{1}{\gamma(y)} d y \\
& =\int_{0}^{\frac{1}{2}} \frac{v_{1}^{2}(0, y)}{\gamma(y)}=\int_{0}^{\frac{1}{2}} \frac{y^{2 \beta}}{y^{\alpha^{-1}}},
\end{aligned}
$$

which implies that $\left\|\nabla v_{1}\right\|_{L^{2}}=\infty$ if $2 \beta-\alpha^{-1} \leq-1$, i.e., if $\alpha \leq \frac{1}{1+2 \beta}$. Thus, for any $\alpha<1$, this shows that $v_{1} \notin V_{1}$ if we choose $\beta>0$ sufficiently small, which leads to a contradiction. Hence the second claim follows.

${ }^{1}$ Strictly speaking this is not necessarily meaningful due to possible lack of regularity of $v_{1}$. However, it is true for smooth functions and therefore one can argue by density. 
Consider for any $\varepsilon \in(0,1)$ and $\lambda>0$ the sets

$$
X_{\lambda, \varepsilon}:=\left\{u \in H_{0}^{1}(\Omega): u(0, y) \geq \lambda y^{\beta} \text { for a.e. } y \in(0, \varepsilon)\right\}
$$

where the inequality has to be understood in the sense of traces. Notice that $\cup_{\lambda>0} X_{\lambda, \varepsilon}$ is dense in $V$ for any $0<\varepsilon<1$. Moreover, if $\beta=\left(\alpha^{-1}-1\right) / 2$, then according to the proof of Theorem 1 it holds that $X_{\lambda, \varepsilon} \subset V \backslash\left(V_{1}+V_{2}\right)$. Hence, Theorem 3 implies that any $e_{0} \in X_{\lambda, \varepsilon}$ leads to a NGC.

In view of Theorem 1, the geometric convergence of the Schwarz method (as APM) does not hold. This is due to results that we discuss in Section 3.

\section{3 'Slow' convergence in the abstract framework of the APM}

Consider an arbitrary Hilbert space $(V,\langle\cdot, \cdot\rangle)$ and two closed subspaces $V_{1}$ and $V_{2}$ such that $V_{1}+V_{2} \neq \overline{V_{1}+V_{2}}$. Denote by $\|\cdot\|$ the norm induced by $\langle\cdot, \cdot\rangle .^{2}$ Does the APM, corresponding to the iteration operator $P_{V_{2}^{\perp}} P_{V_{1}^{\perp}}$, converge geometrically? The answer is negative and given in Theorem 2 .

Theorem 2 (On the geometric convergence of the APM). Let $V_{1}, V_{2} \subset V$ be closed subspaces of a Hilbert space with $\overline{V_{1}+V_{2}}=V$. Let $\|\cdot\|^{\prime}$ be the operator norm induced by $\|\cdot\|$. The following statements are equivalent.

(i) $V_{1}+V_{2}=V$.

(ii) $\left\|P_{V_{2}^{\perp}} P_{V_{1}^{\perp}}\right\|^{\prime}<1$.

(iii) There exists $\theta \in[0,1)$ such that $\forall e_{0} \in V$ and $\forall k \in \mathbb{N}\left\|\left(P_{V_{2}^{\perp}} P_{V_{1}^{\perp}}\right)^{k} e_{0}\right\| \leq \theta^{k}\left\|e_{0}\right\|$.

(iv) For all $e_{0} \in V$ there is $\theta_{e_{0}} \in[0,1)$ such that $\forall k \in \mathbb{N}\left\|\left(P_{V_{2}^{\perp}} P_{V_{1}^{\perp}}\right)^{k} e_{0}\right\| \leq \theta_{e_{0}}^{k}\left\|e_{0}\right\|$.

Proof. The implication from (i) to (ii) is well known; see, e.g., [11, 5]. Clearly (ii) implies (iii), and (iii) implies (iv). It remains to prove that (iv) implies (i). To do so, let $e_{0} \in V$, and denote $e_{k}=\left(P_{V_{2}^{\perp}} P_{V_{1}^{\perp}}\right)^{k} e_{0}$. We observe that (iv) implies that $\lim _{k \rightarrow \infty} e_{k}=0$ and that the series $y:=\sum_{k=0}^{\infty} e_{k}$ is absolutely convergent. Moreover, we have

$$
P_{V_{2}^{\perp}} P_{V_{1}^{\perp}} e_{0}=\left(1-P_{V_{2}}\right)\left(1-P_{V_{1}}\right) e_{0}=e_{0}-P_{V_{2}} P_{V_{1}^{\perp}} e_{0}-P_{V_{1}} e_{0} .
$$

By induction and using that $\lim _{k \rightarrow \infty} e_{k}=0$ and that the series $y:=\sum_{k=0}^{\infty} e_{k}$ converges absolutely, we obtain

$$
\begin{aligned}
e_{0} & =\left(P_{V_{1}}+P_{V_{2}} P_{V_{1}^{\perp}}\right) e_{0}+e_{1}=\left(P_{V_{1}}+P_{V_{2}} P_{V_{1}^{\perp}}\right) \sum_{k=0}^{n} e_{k}+e_{n+1} \\
& =\left(P_{V_{1}}+P_{V_{2}} P_{V_{1}^{\perp}}\right) y \in V_{1}+V_{2} .
\end{aligned}
$$

${ }^{2}$ Notice that we consider here the same notation (namely the symbols $V, V_{1}, V_{2},\langle\cdot, \cdot\rangle$ and $\|\cdot\|)$ used in the other sections to describe a more abstract setting. However, it is clear from the context whether the notation refers to an abstract Hilbert space setting or to the precise domain decomposition setting. 
Since $e_{0} \in V$ was arbitrary, the claim follows.

Theorem 2 implies that, if $V_{1}+V_{2}$ is not closed, then there exists an initial function $e_{0}$ such that the APM sequence $\left(e_{k}\right)_{k \in \mathbb{N}}$ does not converge geometrically. The issue of the rate of convergence of the APM when $V_{1}+V_{2}$ is not closed has first been addressed by Franchetti and Light, who prove in [9] the following result.

Theorem 3 (NGC of the APM). Let $V_{1}, V_{2} \subset V$ be as in Theorem 2 and assume that $V_{1}+V_{2}$ is not closed. Then, for all $e_{0} \in V \backslash\left(V_{1}+V_{2}\right)$ it holds that $\sum_{k=1}^{\infty} \frac{\left\|e_{k}\right\|}{\sqrt{k}}=\infty$. In particular, the convergence is $N G C$.

Theorem 3 states that for any initial function $e_{0} \in V \backslash\left(V_{1}+V_{2}\right)$ the convergence of the APM is much slower than geometric. Moreover, in the same paper, the authors provide an example of a non-closed sum $V_{1}+V_{2}$ leading to ASC. In 1997, Bauschke, Borwein and Lewis proved in [3] that ASC holds whenever $V_{1}+V_{2}$ is not closed. However, Bauschke, Deutsch and Hundal pointed out later in [4] that the proof of this result given in [3] is erroneous, and they give a different approach to obtain the same result:

Theorem 4 (Dichotomy between ASC and non-closedness of $\mathbf{V}_{\mathbf{1}}+\mathbf{V}_{\mathbf{2}}$ ). Let $V_{1}, V_{2} \subset$ $V$ be as in Theorem 2. Then, exactly one of the following two statements holds:

(1) $V_{1}+V_{2}$ is closed. Then the convergence is geometric.

(2) $V_{1}+V_{2}$ is not closed. Then the convergence is arbitrarily slow.

In 2010, Deutsch and Hundal studied ASC for a general class of operators on Banach spaces [7, 8]. Their results include Theorem 4, also in the case of more than two subspaces. Independently, the same results have been proved in 2011 by Badea, Grivaux and Müller [1]. In the same paper it is shown that, if $V_{1}+V_{2}$ is not closed, then, for any positive sequence $\left(f_{n}\right)_{n \in \mathbb{N}}$, the set $\left\{e_{0} \in V:\left\|e_{n}\right\| \geq f_{n}\right.$ for a. e. $\left.n \in \mathbb{N}\right\}$ is dense in $V$.

We have seen that if $V_{1}+V_{2}$ is not closed, then the APM converges arbitrarily slow and the convergence is much slower than geometric at least for any initial vector $e_{0} \in V \backslash\left(V_{1}+V_{2}\right)$, and that the set of all $e_{0}$ leading to ASC is dense in $V$. However, what is the dependence of the convergence rate on the initial vector $e_{0}$ ? Can one characterize the set of all $e_{0}$ leading to geometric convergence? In the papers mentioned above, there are only a few sentences hinting on the dependence of the convergence rate on the starting point $e_{0}$. In [2], Badea and Seifert have shown that one can always find a dense subset $W \subset V$ for which 'super-polynomially fast convergence' holds. However, it seems difficult to characterize such a subset in a concrete example. In the following section, we discuss the dependence of the convergence rate on the starting point $e_{0}$ for the specific example from Section 2. In particular, we provide rigorous results on the regularity that is needed for an initial function $e_{0}$ to lead to geometric convergence, and show that the set of these initial functions is a dense subset of $\mathrm{V}$ if the overlap of the domains is not too rough $(\alpha>1 / 3)$. 


\section{The dependence of the convergence rate on the initial function}

Consider the domain decomposition studied in Section 2 with a non-Lipschitz overlap $D$. Recall also $V=H_{0}^{1}(\Omega)$ and the two subspaces $V_{1}$ and $V_{2}$ whose orthogonal complements are given in (3). For which initial functions $e_{0} \in V$ does the Schwarz method converge geometrically?

Probably the functions that come first to the mind of the reader are the ones in $V$ that vanish on the interface $\Gamma_{1}:=\overline{\partial \Omega_{1} \cap \Omega}$. For these functions, the Schwarz method (2) converges in only one step. Indeed, with $F:=\left\{v \in V: v=0\right.$ on $\left.\partial \Omega_{1} \cap \Omega\right\}$, we see that $\operatorname{ker}\left(P_{V_{2}^{\perp}} P_{V_{1}^{\perp}}\right)=V_{1} \oplus\left(V_{1}^{\perp} \cap V_{2}\right)=V_{1} \oplus\left\{v \in V: v=0\right.$ in $\left.\overline{\Omega_{1}}\right\}=F$, where we used (3). It is not difficult to see that, if $u \notin F$, then the iteration will not yield the exact result after any finite number of iterations. Moreover, $F$ is not the maximal set of functions that lead to geometric convergence. This is clearly shown by Theorem 5 below. To prove it, we need the following lemma.

Lemma 1. Let $V_{1}, V_{2} \subset V$ be as in Theorem 2, and let $W \subset V_{1}+V_{2}$ be a closed subspace which is invariant under $P_{V_{2}^{\perp}} P_{V_{1}^{\perp}}$. Then, there exists $\theta<1$ such that $\left\|\left(P_{V_{2}^{\perp}} P_{V_{1}^{\perp}}\right)^{k} e_{0}\right\| \leq \theta^{k}\left\|e_{0}\right\|$ for all $e_{0} \in W$.

Proof. The result follows by the same arguments used in [11, Theorem I.1].

Theorem 5 (A set of initial functions leading to geometric convergence). Recall the domain decomposition given in Theorem 1 and the corresponding parameter $\alpha$. Consider for an arbitrary $\lambda>0$ the set

$$
W_{\lambda}:=\{v \in V: v(x, y) \leq \lambda y \text { for almost all }(x, y) \in \Omega\}
$$

For all $1>\alpha>1 / 3$, the sets $W_{\lambda}$ are closed subspaces of $V_{1}+V_{2}$ and invariant under $P_{V_{2}^{\perp}} P_{V_{1}^{\perp}}$. Moreover, $\cup_{\lambda>0} W_{\lambda}$ is dense in $V$, and for any $\lambda>0$ there exists $\theta<1$ such that

$$
\left\|\left(P_{V_{2}^{\perp}} P_{V_{1}^{\perp}}\right)^{k} e_{0}\right\| \leq \theta^{k}\left\|e_{0}\right\| \quad \text { for all } e_{0} \in W_{\lambda} .
$$

Proof. Notice that $W_{\lambda}$ are closed subspaces of $V$ and $C_{c}^{\infty}(\Omega) \subset \cup_{\lambda>0} W_{\lambda}$. Hence $\cup_{\lambda>0} W_{\lambda}$ is dense in $V$.

To show that $W_{\lambda} \subset V_{1}+V_{2}$, we define the cut-off function $\eta: \Omega \rightarrow \mathbb{R}$ by

Then, for $(x, y) \in D$ we have

$$
\eta(x, y)= \begin{cases}0 & \text { in } \Omega_{1} \backslash \Omega_{2}, \\ 1 & \text { in } \Omega_{2} \backslash \Omega_{1}, \\ x y^{-\alpha^{-1}} & \text { in } D=\Omega_{1} \cap \Omega_{2} .\end{cases}
$$

$$
|\nabla \eta(x, y)|=\left|\left(y^{-\alpha^{-1}},-\alpha^{-1} x y^{-\alpha^{-1}-1}\right)\right| \leq C(\alpha) y^{-\alpha^{-1}} .
$$

Let now $\lambda>0$ be fixed and let $w \in W_{\lambda}$. Then we claim $\eta w \in V_{1}$ and $(1-\eta) w \in V_{2}$. Using (5) and recalling that $\alpha>1 / 3$, we get 


$$
\begin{aligned}
\|(\nabla \eta) w\|_{L^{2}(\Omega)}^{2} & \leq \int_{D} C(\alpha) \lambda^{2} \frac{y^{2}}{y^{2 \alpha^{-1}}}=C(\alpha) \lambda^{2} \int_{0}^{1} \int_{0}^{y^{\alpha^{-1}}} \frac{y^{2}}{y^{2 \alpha^{-1}}} d x d y \\
& =C(\alpha) \lambda^{2} \int_{0}^{1} \frac{y^{2}}{y^{\alpha^{-1}}} d y=C(\alpha) \lambda^{2} \frac{1}{3-\alpha^{-1}}
\end{aligned}
$$

Noticing $\eta \leq 1$ in $\Omega$, the above estimate shows $\eta w \in V_{1}$ and $(1-\eta) w \in V_{2}$.

Next, we show that $W_{\lambda}$ are invariant under $P_{V_{i}^{\perp}}, i=1,2$. Let $w \in W_{\lambda}$. Then $v:=$ $P_{V_{1}^{\perp}} w$ is the unique function such that $\Delta v=0$ in $\Omega_{1}$ with $v=w$ in $\Omega \backslash \Omega_{1}$. Therefore, since the function $\varphi(x, y)=\lambda y$ is harmonic, the maximum principle implies that $v \leq \varphi$ in $\Omega_{1}$ and clearly also that $v=w \leq \varphi$ in $\Omega \backslash \Omega_{1}$. Hence $v \in W_{\lambda}$. The invariance under $P_{V_{2}^{\perp}}$ is analogous. Therefore, we obtain that $W_{\lambda}$ is invariant under $P_{V_{2}^{\perp}} P_{V_{1}^{\perp}}$. Finally, the geometric convergence follows from Lemma 1.

Theorem 5 says that for $\alpha>1 / 3$ we have geometric convergence for all $e_{0} \in$ $\cup_{\lambda>0} W_{\lambda}$. The restriction $\alpha>1 / 3$ is optimal. To see it, recall the sets $X_{\lambda, \varepsilon}$ defined in (4) and that $X_{\lambda, \varepsilon} \subset V \backslash\left(V_{1}+V_{2}\right)$. Hence, Theorem 3 guarantees that any $e_{0} \in X_{\lambda, \varepsilon}$ leads to a NGC. However, for $\alpha \leq 1 / 3, X_{\lambda, \varepsilon}$ and $W_{\lambda}$ have non-trivial intersections. Therefore, if $\alpha \leq 1 / 3$, then there exists $e_{0} \in W_{\lambda}$, in particular $e_{0} \in W_{\lambda} \cap X_{\lambda, \varepsilon}$, that leads to NGC.

\section{Numerical experiments}

In this section, we present a numerical study of the NGC of the Schwarz method corresponding to the domain decomposition given in Fig. 1. The (monodomain) problem is discretized by linear finite elements using the software Freefem. The discrete meshes for $\Omega_{1} \backslash \Omega_{2}, D$ and $\Omega_{2} \backslash \Omega_{1}$ are obtained by the mesh generator of Freefem where we discretized the boundary components $\Gamma_{A}, \Gamma_{D}, \Gamma_{E}$ and $\Gamma_{F}$ with 10 points and $\Gamma_{B}, \Gamma_{C}, \Gamma_{1}$ and $\Gamma_{2}$ with $10 N$ points with a positive integer $N$. This choice is motivated by the higher accuracy needed close to the singularity point of $\partial D$. The results of our numerical experiments are shown in Fig. 2, where we plot the value $1-\left\|e_{n}\right\| /\left\|e_{n-1}\right\|$ for the iteration count $n=1, \ldots, 2000$. The numerical procedure is stopped only if $\left\|e_{n}\right\|<10^{-16}$ or if the value $1-\left\|e_{n}\right\| /\left\|e_{n-1}\right\|$ becomes too small (or negative). Clearly, if $1-\left\|e_{n}\right\| /\left\|e_{n-1}\right\|$ becomes constant as $n$ grows, then the method reached a geometric convergence regime. On the other hand, if $1-\left\|e_{n}\right\| /\left\|e_{n-1}\right\| \rightarrow 0$ as $n$ grows, then the method converges non-geometrically. Motivated by Theorems 1 and 5, we study the numerical behavior of the Schwarz method for an overlap characterized by $\alpha=\frac{\theta}{2}+\frac{1-\theta}{3}$ for different $\theta$ in $[0,1]$, an initial guess $e_{0} \in W_{1}$, and different $N$. In particular, according to Theorem 5, we expect geometric convergence for any $\theta \in(0,1]$ and NGC for $\theta=0$. In Fig. 2, we see that for $N=10$ the Schwarz method is numerically geometric convergent for $\theta \in[1 / 2,1]$ (solid lines), but not for $\theta<1 / 2$ (dashed lines). However, if one refines the mesh with $N=20$ and $N=30$, then geometric convergence holds also for $\theta=$ 0.4 and $\theta=0.3$. Moreover, for bigger $N$ also the curves for smaller $\theta$ are less steep 

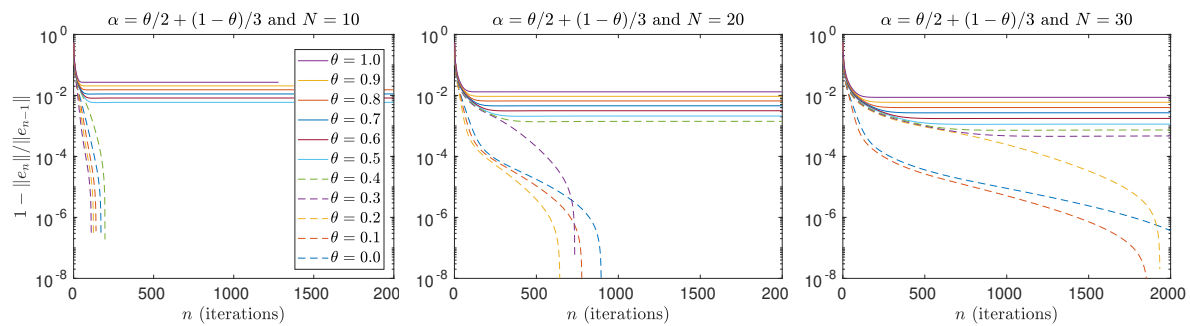

Fig. 2 Convergence behavior of the Schwarz method. The value $1-\left\|e_{n}\right\| /\left\|e_{n-1}\right\|$ is shown for $N=10$ (left), $N=20$ (center), $N=30$ (right).

and show a behavior closer to the proved geometric convergence. Finally, we wish to remark that, according to our experience, a more precise numerical description of the correct theoretical behavior for $\theta$ approaching zero is hard. This is mainly due to the non-Lipschitz overlap, where a correct numerical discretization is not trivial. Therefore, further studies would be needed. These are beyond the scope of this short manuscript, and we hope to consider them in future work.

\section{References}

1. C. Badea, S. Grivaux, and V. Müller, The rate of convergence in the method of alternating projections, Algebra i Analiz 23 (2011), no. 3, 1-30.

2. C. Badea and D. Seifert, Ritt operators and convergence in the method of alternating projections, J. Approx. Theory 205 (2016), 133-148.

3. H.H. Bauschke, J.M. Borwein, and A.S. Lewis, The method of cyclic projections for closed convex sets in Hilbert space, Recent developments in optimization theory and nonlinear analysis (Jerusalem, 1995), Contemp. Math., vol. 204, Amer. Math. Soc., Providence, RI, 1997, pp. 1-38.

4. H.H. Bauschke, F. Deutsch, and H. Hundal, Characterizing arbitrarily slow convergence in the method of alternating projections, Int. Trans. Oper. Res. 16 (2009), no. 4, 413-425.

5. G. Ciaramella and M.J. Gander, Analysis of the parallel Schwarz method for growing chains of fixed-sized subdomains: Part III, ETNA 48 (2018), 201-243.

6. F. Deutsch, Best approximation in inner product spaces, CMS Books in Mathematics/Ouvrages de Mathématiques de la SMC, vol. 7, Springer-Verlag, New York, 2001.

7. F. Deutsch and H. Hundal, Slow convergence of sequences of linear operators I: almost arbitrarily slow convergence, J. Approx. Theory 162 (2010), no. 9, 1701-1716.

8. _ Slow convergence of sequences of linear operators II: arbitrarily slow convergence, J. Approx. Theory 162 (2010), no. 9, 1717-1738.

9. C. Franchetti and W. Light, On the von Neumann alternating algorithm in Hilbert space, J. Math. Anal. Appl. 114 (1986), no. 2, 305-314.

10. I. Halperin, The product of projection operators, Acta Sci. Math. (Szeged) 23 (1962), 96-99.

11. P.L. Lions, On the Schwarz alternating method. I, First International Symposium on Domain Decomposition Methods for Partial Differential Equations (Paris, 1987), SIAM, Philadelphia, PA, 1988, pp. 1-42.

12. J. von Neumann, On rings of operators. Reduction theory, Ann. of Math. (2) 50 (1949), 401485. 